\title{
Homotopy probability theory II
}

\author{
Gabriel C. Drummond-Cole · Jae-Suk Park • \\ John Terilla
}

Received: 25 October 2013 / Accepted: 10 March 2014 / Published online: 6 April 2014

(C) Tbilisi Centre for Mathematical Sciences 2014

\begin{abstract}
This is the second of two papers that introduce a deformation theoretic framework to explain and broaden a link between homotopy algebra and probability theory. This paper outlines how the framework can assist in the development of homotopy probability theory, where a vector space of random variables is replaced by a chain complex of random variables. This allows the principles of derived mathematics to participate in classical and noncommutative probability theory. A simple example is presented.
\end{abstract}

Keywords Probability $\cdot$ Cumulants $\cdot$ Homotopy

Communicated by Jim Stasheff.

This material is based in part upon work supported by the National Science Foundation under Award No. DMS-1004625. This work was supported in part by the Mid-career Researcher Program through NRF funded by the MEST (no. 2010-0000497). Thanks to the Simons Center for Geometry and Physics for providing an excellent working environment.

G. C. Drummond-Cole · J.-S. Park

Center for Geometry and Physics, Institute for Basic Science (IBS), Pohang 790-784,

Republic of Korea

e-mail: gabriel@ibs.re.kr

\section{J.-S. Park}

Department of Mathematics, Pohang University of Science and Technology (POSTECH),

Pohang 790-784, Republic of Korea

e-mail: jaesuk@postech.ac.kr

J. Terilla $(\varangle)$

Department of Mathematics, The Graduate Center and Queens College,

The City University of New York, New York, USA

e-mail: jterilla@qc.cuny.edu 


\section{Mathematics Subject Classification (2000) 55U35 - 46L53 - 60Axx}

\section{Introduction}

This paper is the second of two papers that interprets and utilizes a link between homotopy algebra and probability theory [9] found while studying certain algebraic aspects of quantum field theory [7,8]. In the prequel [3], the authors present a deformation theoretic framework for studying maps between algebras that do not respect structure. The framework for studying maps that do not respect structure applies to probability theory in the following way. Expectation value is a linear map between a vector space $V$ of random variables and the complex numbers that does not respect the product structure on $V$. The failure of the expectation value to respect the products in the space of random variables and the complex numbers can be processed to give an infinite sequence of operations $\kappa_{n}: V^{\otimes n} \rightarrow \mathbb{C}$. This sequence of operations assembles into an $A_{\infty}$ morphism between two trivial $A_{\infty}$ algebras. The main proposition in [3] is that the $A_{\infty}$ morphism obtained via this process coincides with the cumulants of the initial probability space.

In the case that the product of random variables is commutative, there is a similar construction of an infinite sequence of symmetric operations $k_{n}: S^{n} V \rightarrow \mathbb{C}$ which assemble into an $L_{\infty}$ morphism. In the commutative context, the result is that the $L_{\infty}$ morphism coincides with the classical cumulants of the initial probability space.

The framework for studying maps which fail to preserve structure applies just as well when the space of random variables $V$ is replaced by a chain complex $C=(V, d)$. This generalization is pursued in this paper.

The idea of replacing a space of random variables by a chain complex of random variables is not unprecedented. For example, the Batalin-Vilkovisky formalism [1] in quantum field theory involves a differential, called the BV differential, on the space of random variables $[2,7,8]$. In this formalism, expectation value is defined via the ***Feynmann path integral which is a chain map from the space of random variables to $\mathbb{C}[[\hbar]]$. The BV differential provides a way to capture some computational techniques of physicists as homological algebra. This work on homotopy probability theory is about adapting physicists' computational techniques directly to probability theory. The presence of Planck's constant $\hbar$ in quantum field theory, specifically the manner in which the products of observables and the BV operator interact with $\hbar$, makes quantum field theory significantly more complicated than probability theory. One may think of a hierarchy with the proposed homotopy probability theory in the middle.

- Classical and noncommutative probability theory are concerned with the expected value of a vector space of random variables equipped with an associative binary product.

- Homotopy probability theory is concerned with the expected values of a chain complex of random variables equipped with an associative binary product.

- Quantum field theory is concerned with the $\mathbb{C}[[\hbar]]$-valued expected values of a chain complex of random variables, called observables, equipped with an associative binary product satisfying certain subtle algebraic conditions related to $\hbar$. 
In this paper, the idea of working with chain complexes rather than vector spaces is isolated and studied — only the middle theory above is considered. Also, to keep the abstract ideas presented here connected to simple examples, the product of random variables will be assumed to be commutative throughout. So, $L_{\infty}$ algebras, rather than $A_{\infty}$ algebras, are used. The requisite modifications and results contained in [3] are given in Sect. 2.

The reasons that quantum field theory frequently involves a differential have little to do with quantum fields. Rather, the differential is a tool used to handle symmetries by algebraic methods, and this tool can be applied to ordinary probability theory. In much the same way that one may resolve relations in homological algebra by replacing a module with a free resolution, one might extend a space of random variables with relations among expectation values to a larger chain complex of random variables where the relations are encoded in the differential. Then homotopical computations provide new ways to compute invariants of interest, such as joint moments. This idea is illustrated with a toy example in Sect. 4. Of course, there may be several ways for a given space of random variables to be extended to a chain complex of random variables, so care should be taken to understand which quantities are invariant of the original space of random variables and do not depend on choices made in a particular extension. This leads to the concept of homotopy random variables (Definition 7) and their moments and cumulants (Definitions 8 and 9).

Independent of any utilitarian advantages to replacing spaces of random variables by chain complexes of random variables, homotopy theoretic ideas provide attractive structural aspects to probability theory. Cumulants, for example, are interpreted as a homotopy morphism and then tools in homotopy theory, both computational and theoretical, can be brought to bear upon them. As another example, this point of view indicates how to construct a category of probability spaces that is different than previous constructions - there are, for example, many more morphisms. Moreover, once the framework for studying maps that do not respect structure is applied to probability theory, there become ways for the ideas, language, and tools of probability theory to participate in other areas that feature a non-structure preserving map. Period integrals of smooth projective hypersurfaces are one such area where the participation seems to generate some new concepts [6].

The authors would like to thank Tyler Bryson, Joseph Hirsh, Tom LeGatta, and Bruno Vallette for many helpful discussions.

\section{Commutative homotopy probability spaces and $L_{\infty}$ algebras}

Definition 1 A commutative homotopy probability space is a triple $(C, e, a)$, where $C=(V, d)$ is a chain complex over $\mathbb{C}, e$ is a chain map $C \rightarrow(\mathbb{C}, 0)$ (where $\mathbb{C}$ is concentrated in degree 0 ), and $a$ is an associative product $S^{2} V \rightarrow V$. $S V$ denotes the graded symmetric algebra so that $a$ is graded symmetric

$$
a\left(X_{1}, X_{2}\right)=(-1)^{\left|X_{1}\right|\left|X_{2}\right|} a\left(X_{2}, X_{1}\right) .
$$


Remark 1 Note that $a$ is not required to possess any sort of compatibility with $d$ or $e$ (which it will not in interesting examples).

Remark 2 There are multiple easy generalizations to this definition that this paper will not pursue. It is natural to replace $\mathbb{C}$ with a different ground algebra. It is also reasonable to allow $a$ to be any element of hom $(S V, V)$. For example, $a$ might be a non-associative binary product, or a collection of non-binary products.

Definition 2 A morphism of commutative homotopy probability spaces between $(C, e, a)$ and $\left(C^{\prime}, e^{\prime}, a^{\prime}\right)$ is a chain map $f: C \rightarrow C^{\prime}$ that commutes with expectation in the sense that $e^{\prime} f=e$.

Remark 3 Note again that there is no compatibility assumed between $f$ and the products $a$ and $a^{\prime}$.

Basic facts and definitions about $L_{\infty}$ algebras are now recalled. For more details, see [4]. Let $V$ be a graded vector space. Let $S^{n} V$ be the $S_{n}$-invariant subspace of $V^{\otimes n}$ and let $S V=\oplus_{n=1}^{\infty} S^{n} V$. As a direct sum, linear maps from $S V$ to a vector space $W$ correspond to collections of linear maps $\left\{S^{n} V \rightarrow W\right\}_{n=1}^{\infty}$. Also, $S V$ is a coalgebra, free in a certain sense, so that linear maps from a commutative coalgebra $\mathcal{C} \rightarrow V$ are in bijection with the following two sets: $\{$ coalgebra maps from $\mathcal{C}$ to $S V\}$ and $\{$ coderivations from $\mathcal{C}$ to $S V$ \}.

Definition 3 An $L_{\infty}$ algebra is a pair $(V, D)$ where $V$ is a graded vector space and $D: S V \rightarrow S V$ is a degree one coderivation satisfying $D^{2}=0$. An $L_{\infty}$ morphism between two $L_{\infty}$ algebras $(V, D)$ and $\left(V^{\prime}, D^{\prime}\right)$ is a differential coalgebra map $F$ : $(S V, D) \rightarrow\left(S V^{\prime}, D^{\prime}\right)$. In other words, an $L_{\infty}$ map from $(V, D)$ to $\left(V^{\prime}, D^{\prime}\right)$ is a degree zero coalgebra map $F: S V \rightarrow S V^{\prime}$ satisfying $F D=D^{\prime} F$.

Remark 4 Often, the definition of an $L_{\infty}$ algebra involves a degree shift (so according to that convention, a degree one coderivation $D: S V \rightarrow S V$ satisfying $D^{2}=0$ would define an $L_{\infty}$ algebra on the underlying vector space $\left.V[-1]\right)$. For the applications to probability theory proposed in this paper, the conventional degree shift makes the signs much more complicated-it's simpler to eliminate the shift in the definition.

The identification

$$
\operatorname{Coder}(S V, S V) \simeq \prod_{n=1}^{\infty} \operatorname{hom}\left(S^{n} V, V\right)
$$

can be used to give the data of an $L_{\infty}$ algebra. That is, a coderivation $D: S V \rightarrow S V$ can be given by a sequence $\left\{d_{n}\right\}$ of degree one linear maps $d_{n}: S^{n} V \rightarrow V$ on a graded vector space $V$. The condition that $D^{2}=0$ implies an infinite number of relations satisfied by various compositions among the $\left\{d_{n}\right\}$. Likewise, the identification

$$
\operatorname{Coalg}\left(S V, S V^{\prime}\right) \simeq \prod_{n=1}^{\infty} \operatorname{hom}\left(S^{n} V, V^{\prime}\right)
$$

can be used to give the data of an $L_{\infty}$ morphism between $(V, D)$ and $\left(V^{\prime}, D^{\prime}\right)$. That is, a coalgebra map $F: S V \rightarrow S V^{\prime}$ can be given by a sequence $\left\{f_{n}\right\}$ of degree zero 
maps $f_{n}: S^{n} V \rightarrow V^{\prime}$. The condition that $F D=D^{\prime} F$ encodes an infinite number of relations among the $\left\{f_{n}\right\}$, the $\left\{d_{n}\right\}$ and the $\left\{d_{n}^{\prime}\right\}$. So, the identifications in Eqs. (1) and (2) provide two equivalent ways of describing $L_{\infty}$ algebras and morphisms and it is convenient to move between the ways since certain notions or computations are easier to describe in one or the other descriptions of the equivalent data. For example, it is straightforward to compose two differential coalgebra maps $F:(S V, D) \rightarrow$ $\left(S V^{\prime}, D^{\prime}\right)$ and $G:\left(S V^{\prime}, D^{\prime}\right) \rightarrow\left(S V^{\prime \prime}, D^{\prime \prime}\right)$ as $G F:(S V, D) \rightarrow\left(S V^{\prime \prime}, D^{\prime \prime}\right)$ and thus define the composition of $L_{\infty}$ morphisms, but it is more involved to express the components $(g f)_{n}: S^{n} V \rightarrow V^{\prime \prime}$ in terms of the $f_{k}: S^{k} V \rightarrow V^{\prime}$ and $g_{m}: S^{m} V^{\prime} \rightarrow$ $V^{\prime \prime}$.

Construction Let $(V, D)$ be an $L_{\infty}$ algebra with components $d_{n}$. Let $A=(W, d)$ be a commutative differential graded algebra. Then $(V \otimes W)$ can be given a canonical $L_{\infty}$ algebra structure denoted $\left(V_{A}, D_{A}\right)$ whose first component is $d_{1} \otimes \mathrm{id}+\mathrm{id} \otimes d$ and whose $n$th component for $n>1$ are given by

$$
\left(v_{1} \otimes w_{1}, \ldots, v_{n} \otimes w_{n}\right) \mapsto d_{n}\left(v_{1}, \ldots, v_{n}\right) \otimes w_{1} \cdots w_{n}
$$

In particular, if $A=(\mathbb{C}, 0)$ with the usual multiplication, then $\left(V_{A}, D_{A}\right)$ is canonically isomorphic to $(V, D)$.

Definition 4 Let $(V, D)$ and $\left(V^{\prime}, D^{\prime}\right)$ be $L_{\infty}$ algebras. Let $\Omega$ denote the commutative differential graded algebra $\mathbb{C}[t, d t]$, polynomials in a variable $t$ of degree 0 and its differential $d t$ (so in particular $(d t)^{2}=0$ ). An $L_{\infty}$ homotopy $H$ from $(V, D)$ to $\left(V^{\prime}, D^{\prime}\right)$ is an $L_{\infty}$ morphism from $(V, D)$ to $\left(V_{\Omega}^{\prime}, D_{\Omega}^{\prime}\right)$. Given an $L_{\infty}$ homotopy $H$, the evaluation maps $\Omega \rightarrow \mathbb{C}$ for $t=0$ and $t=1$ induce two $L_{\infty}$ morphisms from $(V, D)$ to $\left(V^{\prime}, D^{\prime}\right)$. Call these two $L_{\infty}$ morphisms $f$ and $f^{\prime}$. We say that $H$ is a homotopy between $f$ and $f^{\prime}$.

Remark 5 Homotopy is an equivalence relation on $L_{\infty}$ morphisms from $(V, D)$ to $\left(V^{\prime}, D^{\prime}\right)$. Transitivity is not obvious but can be verified [5].

Remark 6 Homotopy behaves well with respect to composition. That is, if $f$ and $f^{\prime}$ are homotopic $L_{\infty}$ maps $P \rightarrow Q$ and $g$ and $g^{\prime}$ are homotopic $L_{\infty}$ maps $Q \rightarrow R$, then $g \circ f$ and $g^{\prime} \circ f^{\prime}$ are homotopic $L_{\infty}$ maps $P \rightarrow R$. In particular, this is true in the special cases where $f=f^{\prime}$ or when $g=g^{\prime}$.

Note that $L_{\infty}$ structures can be transported via isomorphisms. In particular, if $(V, D)$ is an $L_{\infty}$ algebra and $G: S V \rightarrow S V$ is any degree zero coalgebra isomorphism, then for

$$
D^{G}:=G^{-1} D G
$$

the pair $\left(V, D^{G}\right)$ is again an $L_{\infty}$ algebra and the map $G:\left(S V, D^{G}\right) \rightarrow(S V, D)$ is an $L_{\infty}$ algebra isomorphism: 


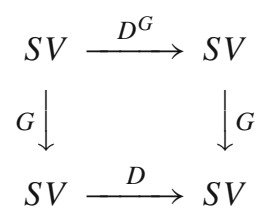

Morphisms and homotopies can be transported as well. If $F$ is an $L_{\infty}$ morphism between $(V, D)$ and $\left(V^{\prime}, D^{\prime}\right)$ and $G: S V \rightarrow S V$ and $G^{\prime}: S V^{\prime} \rightarrow S V^{\prime}$ are coalgebra isomorphisms, then

$$
F^{G, G^{\prime}}:=\left(G^{\prime}\right)^{-1} F G
$$

is an $L_{\infty}$ morphism between the $L_{\infty}$ algebras $\left(V, D^{G}\right)$ and $\left(V^{\prime},\left(D^{\prime}\right)^{G^{\prime}}\right)$. Here's the diagram:

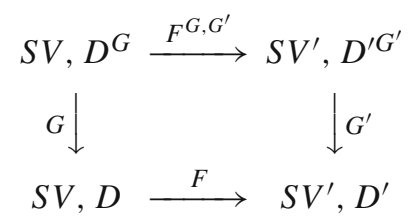

For a homotopy $H: S V \rightarrow S V^{\prime} \otimes \Omega$, the map $G^{\prime}: S V^{\prime} \rightarrow S V^{\prime}$ must be modified to be a coalgebra isomorphism from $S\left(V^{\prime} \otimes \Omega\right)$ to $S\left(V^{\prime} \otimes \Omega\right)$ but by abuse of notation the transferred homotopy $S V \rightarrow S\left(V^{\prime} \otimes \Omega\right)$ will still be referred to as $H^{G, G^{\prime}}$.

Lemma 1 Let $(V, 0)$ be a trivial $L_{\infty}$ algebra. If $f$ and $f^{\prime}$ are homotopic $L_{\infty}$ morphisms $(V, 0) \rightarrow(\mathbb{C}, 0)$, then $f=f^{\prime}$.

Proof Let $H$ be a homotopy between $f$ and $f^{\prime}$. Then $H$ is an $L_{\infty}$ morphism from $(V, 0)$ to $\Omega$. That is, $H$ is a coalgebra map $S V \rightarrow S \Omega$ satisfying $d H=0$. In particular, each of the constituent maps $S^{n} V \rightarrow \Omega$ must have closed image in $\Omega$, so they must actually land in $\mathbb{C} \oplus d t \mathbb{C}[t, d t]$. This means that the evaluation of $H$ at 0 and at 1 yield the same map to $\mathbb{C}$, so $f$ and $f^{\prime}$ coincide.

\section{Expectation, moments, and cumulants}

Notation Let $(C, e, a)$ be a commutative homotopy probability space, with $C=$ $(V, d)$. Then $(V, d)$ and $(\mathbb{C}, 0)$ are $L_{\infty}$ algebras with vanishing higher maps and $e$ is an $L_{\infty}$ morphism from $(V, d)$ to $(\mathbb{C}, 0)$. Let a denote the isomorphism of coalgebras $S V \rightarrow S V$ whose $n$th component $S^{n} V \rightarrow V$ is repeated multiplication using $a$. Let $\mathbf{a}^{\prime}$ denote the isomorphism of coalgebras $S \mathbb{C} \rightarrow S \mathbb{C}$ whose $n$th component $S^{n} \mathbb{C} \rightarrow \mathbb{C}$ is repeated complex multiplication. The lowest component of $\mathbf{a}$ and $\mathbf{a}^{\prime}$ are the identity.

Remark 7 In the case of not necessarily commutative homotopy probability theory, where $a$ is not necessarily commutative and $L_{\infty}$ is replaced throughout with $A_{\infty}$, the product $a$ can be extended as a coderivation on $T V=\oplus_{n=1}^{\infty} V^{\otimes n}$. The coalgebra isomorphism a, whose components $V^{\otimes n} \rightarrow V$ are repeated multiplication using $a$, is the exponential of the extended coderivation [3]. In the commutative world the 
map a $: S V \rightarrow S V$ is defined to be the coalgebra isomorphism whose components $S^{n} V \rightarrow V$ are repeated multiplications using $a$, but the coalgebra isomorphism a is not the exponential of the coderivation lift of of $a$.

Definition 5 Let $(C, e, a)$ be a commutative homotopy probability space with $C=$ $(V, d)$. The total moment of $(C, e, a)$ is the $L_{\infty}$ morphism

$$
M:=e^{\mathbf{a}, \text { id }}
$$

from $\left(V, D^{\mathbf{a}}\right)$ to $(\mathbb{C}, 0)$. The $n$th moment map $m_{n}$ is the $n$th component of the total moment $e^{\mathbf{a}, \text { id }}$ which is a map $m_{n}: S^{n} V \rightarrow \mathbb{C}$.

Definition 6 Let $(C, e, a)$ be a commutative homotopy probability space with $C=$ $(V, d)$. The total cumulant of $(C, e, a)$ is the $L_{\infty}$ morphism

$$
K:=e^{\mathbf{a}, \mathbf{a}^{\prime}}
$$

from $\left(V, D^{\mathbf{a}}\right)$ to $(\mathbb{C}, 0)$. The $n t h$ cumulant $k_{n}$ is the $n$th component of the total cumulant $e^{\mathbf{a}, \mathbf{a}^{\prime}}$ which is a map $k_{n}: S^{n} V \rightarrow \mathbb{C}$.

Moments and cumulants of a commutative homotopy probability space are homotopy invariant.

Proposition 1 Let $C$ be a chain complex equipped with a product a. Let e and $e^{\prime}$ be homotopic chain maps $C \rightarrow(\mathbb{C}, 0)$. Then the total moment $M$ of the commutative homotopy probability space $(C, e, a)$ is homotopic to the total moment $M^{\prime}$ of the commutative homotopy probability space $\left(C, e^{\prime}, a\right)$. Also, the total cumulant $K$ of the commutative homotopy probability space $(C, e, a)$ is homotopic to the total cumulant $K^{\prime}$ of the commutative homotopy probability space $\left(C, e^{\prime}, a\right)$.

Proof The transferred homotopy $H^{\text {a,id }}$ is a homotopy between the total moments. The transferred homotopy $H^{\mathbf{a}, \mathbf{a}^{\prime}}$ is a homotopy between the total cumulants.

In applications, one would like to evaluate moments and cumulants on random variables and obtain numbers. Direct application of moments and cumulants yield numbers that are not homotopy invariant. Homotopy random variables are now introducedthey have joint moments and cumulants that are homotopy invariant.

Definition 7 A collection of homotopy random variables $\left(X_{1}, \ldots, X_{n}\right)$ in a commutative homotopy probability space $(C, e, a)$ is the homotopy class of an $L_{\infty}$ map $\left(\mathbb{C}^{n}, 0\right) \rightarrow\left(V, D^{\mathbf{a}}\right)$. The various factors of $\mathbb{C}^{n}$ may be in different degrees. When $n=1$, we call such a collection a homotopy random variable.

Example 1 The simplest kind of homotopy random variable is an $L_{\infty}$ morphism $X$ : $(\mathbb{C}, 0) \rightarrow\left(V, D^{\mathbf{a}}\right)$ whose only nonzero component is a map $\mathbb{C} \rightarrow V$. Such a map is determined by $1 \mapsto x$ for an element $x \in V$. Not any choice of element $x \in V$ will define a homotopy random variable. The condition that $X$ be an $L_{\infty}$ morphism is that $D^{\mathbf{a}} X=0$, which encodes an infinite collection of conditions on $x$. Namely, 
$d x=0, d_{2}^{\mathbf{a}}(x, x)=0$, etc... More generally, a single homotopy random variable $X$ will have components $S^{k} \mathbb{C} \rightarrow V$, each of which is given by a map $S^{k} 1 \mapsto x_{k}$ for some $x_{k} \in V$. So, an arbitrary single random variable can be thought of as a sequence of elements $\left\{x_{k} \in V\right\}$. Note that every closed element $x \in V$ gives rise to a homotopy random variable by transport. That is, if $x \in V$ satisfies $d x=0$, then $1 \mapsto x$ defines a chain map $f:(\mathbb{C}, 0) \rightarrow(V, d)$. Such a chain map is an $L_{\infty}$ morphism $F:(\mathbb{C}, 0) \rightarrow(V, D)$. The transport of $F$ to an $L_{\infty}$ map $F^{\text {id, a }}:(\mathbb{C}, 0) \rightarrow\left(V, D^{\mathbf{a}}\right)$ is a homotopy random variable. Note that not all homotopy random variables arise as the transport of chain maps; an $L_{\infty}$ morphism $X:(\mathbb{C}, 0) \rightarrow\left(V, D^{\mathbf{a}}\right)$ can be transported back to give $L_{\infty}$ morphisms $X^{\mathrm{id},(\mathbf{a})^{-1}}:(\mathbb{C}, 0) \rightarrow(V, D)$, but this map may not only be a chain map, there may be nonzero components $S^{k} \mathbb{C} \rightarrow V$ for $k>1$.

Example 2 The simplest kind of collection of homotopy random variables is an $L_{\infty}$ morphism $\left(X_{1}, \ldots, X_{n}\right):\left(\mathbb{C}^{n}, 0\right) \rightarrow\left(V, D^{\mathbf{a}}\right)$ whose only nonzero component is a map $\mathbb{C}^{n} \rightarrow V$. Such a morphism is determined by the images of the $i$ th standard basis vectors of $\mathbb{C}^{n}$

$$
b_{i} \mapsto x_{i}
$$

for $i=1, \ldots, n$. So, such a collection of homotopy random variables is determined by an ordered collection of elements $x_{1}, \ldots, x_{n} \in V$. As in the case of a single homotopy random variable, not every collection of elements $\left\{x_{1}, \ldots, x_{k}\right\}$ will define a homotopy random variable and an arbitrary collection of homotopy random variables will generally have nonzero components $S^{k} \mathbb{C}^{n} \rightarrow V$ for $k>1$. As in the case of a single homotopy random variable, finite collections of closed elements in $V$ define chain maps $f:(\mathbb{C}, 0) \rightarrow(V, d)$ and can be transported to give collections of homotopy random variables. Not all collections of homotopy random variables arise this way.

Remark 8 Given a collection of homotopy random variables $\left(X_{1}, \ldots, X_{n}\right)$, one can obtain individual homotopy random variables $X_{i}$ by precomposing the inclusion of $\mathbb{C}$ into $\mathbb{C}^{n}$ in the $i$ th factor. It is not true in general that given a set $\left\{X_{i}\right\}_{i=1, \ldots, n}$ of random variables one can meaningfully generate a collection of homotopy random variables $\left(X_{1}, \ldots, X_{n}\right)$.

Definition 8 Let $(C, e, a)$ be a commutative homotopy probability space. The joint moment $M\left(X_{1}, \ldots, X_{n}\right)$ of the collection of homotopy random variables $\left(X_{1}, \ldots, X_{n}\right)$ is defined to be the $L_{\infty}$ morphism

$$
M\left(X_{1}, \ldots, X_{n}\right)=M \circ f
$$

where $f:\left(\mathbb{C}^{n}, 0\right) \rightarrow\left(V, D^{\mathbf{a}}\right)$ is any representative of the collection of homotopy random variables $\left(X_{1}, \ldots, X_{n}\right)$ and $M$ is the total moment of $(C, e, a)$. The $r$ th joint moment of a collection of random variables is the $r$ th component of the joint moment $M\left(X_{1}, \ldots, X_{n}\right)$ and so is a map $m_{r}\left(X_{1}, \ldots, X_{n}\right): S^{r} \mathbb{C}^{n} \rightarrow \mathbb{C}$.

Definition 9 Let $(C, e, a)$ be a commutative homotopy probability space. The joint cumulant $C\left(X_{1}, \ldots, X_{n}\right)$ of the collection of homotopy random variables 
$\left(X_{1}, \ldots, X_{n}\right)$ is defined to be the $L_{\infty}$ morphism

$$
K\left(X_{1}, \ldots, X_{n}\right)=K \circ f
$$

where $f:\left(\mathbb{C}^{n}, 0\right) \rightarrow\left(V, D^{\mathbf{a}}\right)$ is any representative of the collection of homotopy random variables $\left(X_{1}, \ldots, X_{n}\right)$ and $K$ is the total cumulant of $(C, e, a)$. The $r$ th joint cumulant of a collection of random variables is the $r$ th component of the joint cumulant $C\left(X_{1}, \ldots, X_{n}\right)$ and so is a map $k_{r}\left(X_{1}, \ldots, X_{n}\right): S^{r} \mathbb{C}^{n} \rightarrow \mathbb{C}$.

Lemma 2 Joint moments and joint cumulants are well-defined.

Proof Let $f$ and $f^{\prime}$ be two representatives of the homotopy class $\left(X_{1}, \ldots, X_{n}\right)$. Then $M \circ f$ and $M \circ f^{\prime}$ are homotopic $L_{\infty}$ maps from $\left(\mathbb{C}^{n}, 0\right)$ to $(\mathbb{C}, 0)$. By Lemma 1 , these two maps coincide. The compositions $K \circ f$ and $K \circ f^{\prime}$ coincide for the same reason.

Remark 9 The $L_{\infty}$ structure $D^{\mathbf{a}}$ does not depend on $e$. A collection of homotopy random variables could be defined for a pair $(C, a)$ without reference to an expectation. In particular, a collection of homotopy random variables $\left(X_{1}, \ldots, X_{n}\right)$ for a commutative homotopy probability space $(C, e, a)$ is also a collection of homotopy random variables for a commutative homotopy probability space $\left(C, e^{\prime}, a\right)$.

Proposition 2 Let $C$ be a chain complex equipped with a product a. Let e and $e^{\prime}$ be homotopic chain maps $C \rightarrow(\mathbb{C}, 0)$. Let $\left(X_{1}, \ldots, X_{n}\right)$ be a collection of homotopy random variables of the commutative homotopy probability spaces $(C, e, a)$ and $\left(C, e^{\prime}, a\right)$. Then the joint moments and joint cumulants coincide. That is,

$$
m_{r}\left(X_{1}, \ldots, X_{n}\right)=m_{r}^{\prime}\left(X_{1}, \ldots, X_{n}\right)
$$

and

$$
k_{r}\left(S_{1}, \ldots, X_{n}\right)=k_{r}^{\prime}\left(X_{1}, \ldots, X_{n}\right)
$$

Proof Cumulants evaluated on a collection of homotopy random variables constitute $L_{\infty}$ morphisms from $\left(\mathbb{C}^{n}, 0\right) \rightarrow(\mathbb{C}, 0)$. The proposition shows that in the case in question, two such $L_{\infty}$ maps are homotopic. By Lemma 1, they in fact coincide.

To summarize, a collection of random variables $\left(X_{1}, \ldots, X_{n}\right)$ is a homotopy class of $L_{\infty}$ maps $\left(\mathbb{C}^{n}, 0\right) \rightarrow\left(V, D^{\mathbf{a}}\right)$. The joint moments and joint cumulants of a collection of homotopy random variables $e\left(X_{1}, \ldots, X_{n}\right)$ are defined to be the compositions of $L_{\infty}$ morphisms

$$
\begin{aligned}
& \left(\mathbb{C}^{n}, 0\right) \stackrel{\left(X_{1}, \ldots, X_{n}\right)}{\longrightarrow}\left(V, D^{\mathbf{a}}\right) \stackrel{M}{\rightarrow}(\mathbb{C}, 0) \\
& \left(\mathbb{C}^{n}, 0\right) \stackrel{\left(X_{1}, \ldots, X_{n}\right)}{\longrightarrow}\left(V, D^{\mathbf{a}}\right) \stackrel{K}{\rightarrow}(\mathbb{C}, 0)
\end{aligned}
$$

These compositions are well defined. They are independent of the representative of $\left(X_{1}, \ldots, X_{n}\right)$ and only depend on the homotopy class of the expectation value chain 
map $e:(V, d) \rightarrow(\mathbb{C}, 0)$. As an $L_{\infty}$ map $\left(\mathbb{C}^{n}, 0\right) \rightarrow(\mathbb{C}, 0)$, the joint moments and joint cumulants of a collection of homotopy random variables $e\left(X_{1}, \ldots, X_{n}\right)$ have component maps

$$
m_{r}: S^{r}\left(\mathbb{C}^{n}\right) \rightarrow \mathbb{C} \text { and } k_{r}: S^{r}\left(\mathbb{C}^{n}\right) \rightarrow \mathbb{C}
$$

which are comparable to the joint moments and cumulants of a collection of random variables in classical probability theory. In the special case that a linear map $\mathbb{C}^{n} \rightarrow V$ defined by $b_{i} \mapsto x_{i}$ does in fact define an $L_{\infty}$ morphism, as in Example 2, then the joint moment map is given by

$$
\begin{aligned}
S^{r} \mathbb{C}^{n} & \rightarrow \mathbb{C} \\
\left(b_{i_{1}}, \ldots, b_{i_{r}}\right) & \mapsto e\left(x_{i_{1}} \cdots x_{i_{r}}\right)
\end{aligned}
$$

and the joint cumulant map is given by

$$
\begin{aligned}
S^{r} \mathbb{C}^{n} & \rightarrow \mathbb{C} \\
\left(b_{i_{1}}, \ldots, b_{i_{r}}\right) & \mapsto k_{r}\left(x_{i_{1}}, \ldots, x_{i_{r}}\right)
\end{aligned}
$$

where $k_{r}: S^{k} V \rightarrow \mathbb{C}$ is the $r$ th cumulant of $(C, e, a)$.

\section{The Gaussian}

The Gaussian in one variable provides a toy example of how one might extend an algebra of random variables to a chain complex of random variables. The classical Gaussian in one variable is a classical probability space $(V, e, a)$ defined as follows: The space of random variables $V$ is the space of polynomials in one variable, concentrated in degree zero, the expectation $e(p(x))$ of a random variable is given by

$$
e(p(x))=\frac{\int_{\mathbb{R}} p(x) e^{-\frac{x^{2}}{2}} d x}{\int_{\mathbb{R}} e^{-\frac{x^{2}}{2}} d x},
$$

and the product $a: S^{2} V \rightarrow V$ is the ordinary product of polynomials.

Now, we construct a related homotopy probability space $(C, e, a)$. The idea behind the chain complex $(V, d)$ is to add new odd, nilpotent, random variables whose image under the differential comprises the kernel of the expectation value $e$. Let $V=V^{0} \oplus V^{1}$ be the free commutative algebra on two generators $x$ of degree 0 and $\eta$ of degree -1 . The product $a: V \otimes V \rightarrow V$ is the free graded commutative product. A typical element of $V$ has the form $p+q \eta$ for two polynomials $p$ and $q$ in the variable $x$. The $p$ term has degree zero and the $q \eta$ term has degree -1 . The product is determined by the fact that $\eta$ as an element of degree -1 squares to zero. So

$$
(p+q \eta)(r+s \eta)=p r+(p s+q r) \eta .
$$

Define the differential $d: V \rightarrow V$ by

$$
d(p+q \eta)=\frac{d q}{d x}-x q .
$$


Define the expectation $e: V \rightarrow \mathbb{C}$ by

$$
e(p+q \eta)=\frac{\int_{\mathbb{R}} p e^{-\frac{x^{2}}{2}} d x}{\int_{\mathbb{R}} e^{-\frac{x^{2}}{2}} d x}
$$

Since

$$
\begin{aligned}
e(d(p+q \eta)) & =e\left(\frac{d q}{d x}-x q\right) \\
& =\frac{1}{\int_{\mathbb{R}} e^{-\frac{x^{2}}{2}} d x} \int_{\mathbb{R}}\left(\frac{d q}{d x}-x q\right) e^{-\frac{x^{2}}{2}} d x \\
& =\frac{1}{\int_{\mathbb{R}} e^{-\frac{x^{2}}{2}} d x} \int_{\mathbb{R}} \frac{d}{d x}\left(q e^{-\frac{x^{2}}{2}}\right) d x \\
& =0
\end{aligned}
$$

the expectation defines a chain map $e:(V, d) \rightarrow(\mathbb{C}, 0)$. So if two polynomials represent the same homology class in $C$, they have the same expectation. In fact, the converse is true: two polynomials have the same expectation if and only if they represent the same homology class in $C$. So, $d$ encodes all the relations among expectations and to compute the expectation of any one of the original random variables, it suffices to know the homology class of the random variable and the map $e$ on homology. Despite this fact, the map $e$ on homology retains almost no information of the original one variable Gaussian because $e$ on homology has no information about the product structure on the space of random variables. To illustrate this point and the thinking behind homotopy random variables, rephrase things in terms of homotopies of maps.

If $f=f(x)$ and $g=g(x)$ are polynomials that represent the same homology class in $(V, d)$, then the maps $1 \mapsto f$ and $1 \mapsto g$ define homotopic chain maps $(\mathbb{C}, 0) \rightarrow$ $(V, d)$. The corresponding probability theory statement is that their expectations agree $e(f)=e(g)$. However, the moments of $f$ and $g$ will not agree in general, for example $e\left(f^{2}\right)$ and $e\left(g^{2}\right)$ need not agree. If, however, the maps $1 \mapsto f$ and $1 \mapsto g$ define homotopic $L_{\infty}$ maps $(\mathbb{C}, 0) \rightarrow\left(V, D^{\mathbf{a}}\right)$, then not only will their expectations agree, but all of their moments will agree as well. This can be verified explicitly.

First, the $L_{\infty}$ structure $D^{\mathbf{a}}$ on $(V, d)$ can be computed. Recall $D^{\mathbf{a}}$ is the $L_{\infty}$ structure on $(V, d)$ transported by the coalgebra automorphism a $: S V \rightarrow S V$ determined by the product $a: V \otimes V \rightarrow V$ of polynomials:

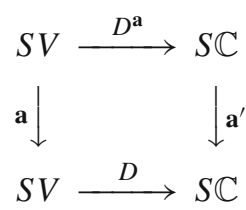

The map $D: S V \rightarrow S \mathbb{C}$ along the bottom is the map $d: V \rightarrow \mathbb{C}$ lifted as a coderivation. Let $d_{n}^{\text {a }}: S^{n} V \rightarrow \mathbb{C}$ denote the components of $D^{\mathbf{a}}$. One way to compute 
$d_{n}$ is to trace an element of $S^{n} V$ from the top left to the bottom right along the two paths in the diagram above and compare the part that lands in $\mathbb{C} \subset S \mathbb{C}$. For $n=1$, this comparison gives $d_{1}^{\mathbf{a}}=d$. For $n=2$, following the diagram along the top and right yields

$$
\begin{aligned}
\left(v_{1}, v_{2}\right) & \mapsto d_{2}^{\mathbf{a}}\left(v_{1}, v_{2}\right)+\left(d\left(v_{1}\right), v_{2}\right)+(-1)^{\left|v_{1}\right|}\left(v_{1}, d\left(v_{2}\right)\right) \\
& \mapsto d_{2}^{\mathbf{a}}\left(v_{1}, v_{2}\right)+d\left(v_{1}\right) v_{2}+(-1)^{\left|v_{1}\right|} v_{1} d\left(v_{2}\right)
\end{aligned}
$$

and along the left and bottom yields

$$
\left(v_{1}, v_{2}\right) \mapsto v_{1} v_{2} \mapsto d\left(v_{1} v_{2}\right)
$$

Equating these and solving for $d_{2}^{\mathbf{a}}$ gives

$$
d_{2}^{\mathbf{a}}\left(v_{1}, v_{2}\right)=d\left(v_{1} v_{2}\right)-d\left(v_{1}\right) v_{2}-(-1)^{\left|v_{1}\right|} v_{1} d\left(v_{2}\right)
$$

Therefore

$$
\begin{aligned}
d_{2}^{\mathbf{a}}(p+q \eta, r+s \eta)= & d(p r+p s \eta+q r \eta)-d(p+q \eta)(r+s \eta) \\
& -p d(r+s \eta)+q \eta d(r+s \eta) \\
= & \frac{d p}{d x} s+q \frac{d r}{d x}-\frac{d q}{d x} s \eta+q \frac{d s}{d x} \eta .
\end{aligned}
$$

The higher components $d_{k}^{\mathbf{a}}=0$ for $k \geq 3$. To see this, it is helpful to notice that

$$
d=\frac{\partial^{2}}{\partial x \partial \eta}-x \frac{\partial}{\partial \eta}
$$

is a degree $\leq 2$ differential operator. Use the diagram as in the description of $d_{2}^{\text {a }}$ to express $d_{3}^{\mathbf{a}}\left(v_{1}, v_{2}, v_{3}\right)$ as the difference of $d\left(v_{1} v_{2} v_{3}\right)$ and a sum of three terms of the form $d_{2}^{\mathbf{a}}\left(v_{i}, v_{j}\right) v_{k}$ and three terms of the form $d\left(v_{i}\right) v_{j} v_{k}$ to check that $d_{3}^{\mathbf{a}}\left(v_{1}, v_{2}, v_{3}\right)=$ 0 . Then, assuming that $d_{n}^{\mathbf{a}}=0$ for $3 \leq n<k$, we get that

$$
\begin{aligned}
d_{k}^{\mathbf{a}}\left(v_{1}, \ldots, v_{k}\right)= & d\left(v_{1} \cdots v_{k}\right) \\
& -\sum d_{2}^{\mathbf{a}}\left(v_{i} v_{j}\right) v_{1} \cdots \widehat{v_{i}} \cdots \widehat{v_{j}} \cdots v_{k}-\sum v_{1} \cdots d\left(v_{i}\right) \cdots v_{k}
\end{aligned}
$$

which vanishes.

The $L_{\infty}$ algebra $\left(V, D^{\mathbf{a}}\right)$ in this case is a differential graded Lie algebra, the bracket defined by $d_{2}^{\text {a }}$ may be familiar as the Poisson bracket defined on the polynomial functions defined on an odd symplectic vector space. Since both $d(f)=0$ and $d_{2}^{\text {a }}(f, f)=0$ for a polynomial $f=f(x)$, the map $1 \mapsto f$ is an $L_{\infty}$ map $(\mathbb{C}, 0) \rightarrow\left(V, D^{\mathbf{a}}\right)$. Because joint moments of homotopy random variables are well defined, if two maps $1 \mapsto f$ and $1 \mapsto g$ are homotopic $L_{\infty}$ maps then $e\left(f^{n}\right)=e\left(g^{n}\right)$ for all $n$. 
As a final computation, let $X$ be the homotopy random variable defined by $1 \mapsto x$. The joint moment of $X$ is an $L_{\infty}$ morphism $M X:(\mathbb{C}, 0) \rightarrow\left(V, D^{\mathbf{a}}\right) \rightarrow(\mathbb{C}, 0)$ where the first map is $X$ and the second is $M:\left(V, D^{\mathbf{a}}\right) \stackrel{M}{\rightarrow}(\mathbb{C}, 0)$. The components of $M X$ are maps $m_{k}: S^{k} \mathbb{C} \rightarrow \mathbb{C}$ given by $S^{k} 1 \mapsto e\left(x^{k}\right)$. As mentioned earlier, the expectation of any one random variable, such as $e\left(x^{k}\right)$, can be computed by understanding the homology class of the random variable and the map $e$ in homology. Here, the homology of $(V, d)$ is one dimensional spanned by the class of 1 and

$$
e(1)=\frac{\int_{\mathbb{R}} e^{-\frac{x^{2}}{2}} d x}{\int_{\mathbb{R}} e^{-\frac{x^{2}}{2}} d x}=1 .
$$

That determines $e$ on homology. To determine the homology class of $x^{k}$, note, $d(-\eta)=$ $x \Rightarrow[x]=[0]$ and for $n>1, d\left(x^{n-1} \eta\right)=(n-1) x^{n-2}-x^{n} \Rightarrow\left[x^{n}\right]=(n-1)\left[x^{n-2}\right]$. So $\left[x^{k}\right]=[0]$ if $k$ is odd and $\left[x^{2 j}\right]=[(2 j-1) ! !]$ if $k=2 j$ is even. Thus, the components of $M X$, where $X$ is the homotopy random variable defined by $1 \mapsto x$ and $M$ is the total joint moment of the Gaussian, are maps $m_{k}: S^{k} \mathbb{C} \rightarrow \mathbb{C}$ given by

$$
m_{k}\left(S^{k} 1\right)= \begin{cases}0 & \text { if } k \text { is odd } \\ (2 j-1) ! ! & \text { for } k=2 \mathrm{j} \text { even }\end{cases}
$$

\section{References}

1. Batalin, I., Vilkovisky, G.: Gauge algebra and quantization. Phys. Lett. B 102, 27-31 (1981)

2. Costello, K.: Renormalization and Effective Field Theory. AMS (2011)

3. Drummond-Cole, G.-C., Park, J.-S., Terilla, J.: Homotopy probability theory I. J. Homotopy Relat. Struct. 1-11 (2013). doi:10.1007/s40062-013-0067-y

4. Loday, J.-L., Vallette, B.: Algebraic Operads. Springer, Berlin (2012)

5. Markl, M.: Deformation Theory of Algebras and Their Diagrams. American Mathematical Society, Providence (2012)

6. Park, J., Park, J.-S.: Period integrals of smooth projective hypersurfaces and homotopy Lie algebras 2013. arXiv: 1310.6710

7. Park, J.-S.: Algebraic principles of quantum field theory I: foundation and an exact solution of BV QFT 2011. arXiv: 1101.4414

8. Park, J.-S.: Algebraic principles of quantum field theory II: quantum coordinates and WDVV equation 2011. arXiv: 1102.1533

9. Park, J.-S.: Homotopical probability space. In: Lecture at CUNY Graduate Center (2011) 